\title{
None of the Above
}

National Cancer Institute

\section{Source}

National Cancer Institute. None of the Above. NCI Thesaurus. Code C133298.

None of the choices above are suitable. 